\title{
Fluorescent Nucleoside Analogues as DNA Probes
}

\author{
Mary E. Hawkins
}

\subsection{Introduction}

The availability of a large selection of fluorescent probes has revolutionized our approaches to studying biochemical reactions and interactions. These probes have become so commonly used and important to many areas of research that it is hard to imagine working without them. This chapter will focus on a more recently developed subclass of DNA probes, nucleoside analogues, which are different from conventional probes in ways that provide opportunities for much more direct approaches in DNA research. In particular, the focus will be on these analogues used as probes incorporated into DNA. The source of their behavioral differences may not be apparent at first glance. An understanding of the differences between the carbon linker type fluorophores and the nucleoside analogues, and their physical relationships within the DNA will make this clear.

The value and usefulness of the rich variety of fluorescent probes currently available is indisputable. They are, however, poorly suited for the type of experiments defined in this chapter. Because the majority of fluorophores are larger than and structurally dissimilar to purines or pyrimidines, they must be placed on a linker arm at some distance from the site of interest on the DNA. While this placement allows them to be used without perturbing the system, in many cases it removes them from the subtle interactions which are often the target of such investigations.

The nucleoside analogues discussed within this chapter include only those probes that incorporate into an oligonucleotide through a deoxyribose linkage, are formulated as phosphoramidites to allow site-selective insertion, and participate in base-stacking (and sometimes base-pairing) interactions within the DNA. It is the base-stacking and base-pairing features that allow us to monitor subtle changes in DNA structure, binding, and composition. Probes that fit these require-

Mary E. Hawkins - Pharmacology and Experimental Therapeutics Section, National Cancer Institute, Bethesda, Maryland 20892.

Topics in Fluorescence Spectroscopy, Volume 7: DNA Technology, edited by Joseph R. Lakowicz, Kluwer Academic/Plenum Publishers, New York, 2003 
ments exhibit dramatic fluorescence changes in part due to base-stacking or basepairing interactions. It has been demonstrated experimentally that subtle changes that disrupt base stacking can clearly be monitored through changes in fluorescence intensity. ${ }^{1-4}$ In many cases, the products of a reaction do not need to be separated prior to analysis, a definite advantage over tagging methods.

In monitoring binding through anisotropy measurements, these analogues display relatively little movement not associated with the motion of the DNA. ${ }^{5}$ In the case of conventionally attached probes, flexibility of linker arms allows movement of the fluorophore in ways that are independent of the movement of the DNA being studied. This higher level of independent motion leads to more complex results.

The ideal is to have nucleoside analogues that are highly fluorescent and structurally as similar as possible to native nucleosides. It is preferable to have a probe with fluorescence properties, such as excitation and emission maxima, that differ from that of native DNA. Additionally, the probe must be stable, soluble, and fluorescent in an aqueous environment and at $\mathrm{pH}$ levels tolerated by enzyme activities and DNA interactions. The ability to place the probe in an oligonucleotide site-specifically using an automated DNA synthesizer is also very helpful.

A new group of nucleoside analogues based on pteridine structures is currently under development which will add to existing fluorescent nucleoside analogues including 2-aminopurine and ethenoadenosine phosphoramidites. The range of structure and fluorescence characteristics encompassed by these probes will help to expand the different types of research that can be done utilizing them. Examples are provided within this chapter detailing applications of fluorescent nucleoside analogues using the pteridine probes. A brief discussion of 2-AP and ethenoadenosine is also included. Some probes being offered commercially as fluorescent nucleoside analogues will not be included, since in many cases the fluorescent moiety is not positioned in the same relationship within the oligonucleotide.

This chapter will define and give examples of the various ways that the subtle relationship between the nucleoside analogue probes and the DNA can be used to observe minute changes within the DNA, often in a real time format. Although some information will be given about fluorescent analogues other than the pteridines, this is by no means a complete listing or a comprehensive review of the subject.

\subsection{Pteridine Nucleoside Analogues}

\subsubsection{Background}

Pteridines are a class of bicyclic planar compounds, some of which are highly fluorescent and structurally similar to purines. Initially 18 pteridine nucleosides 


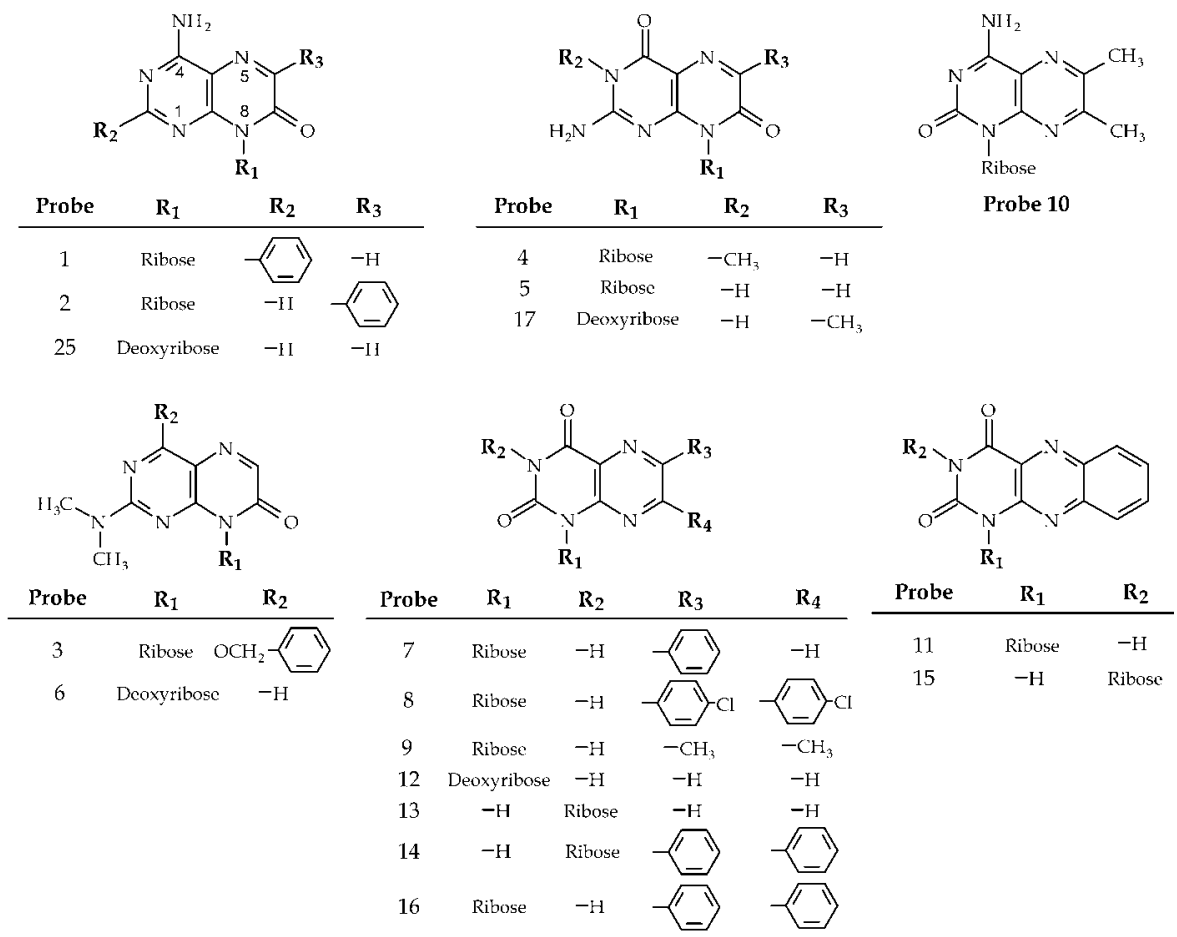

Figure 5.1. Chemical structures of the 18 pteridine nucleoside analogues. Compounds are grouped according to similarities in their chemical structures. The 7 pteridine compounds in the top row were selected for more detailed study based on their favorable $\mathrm{Q}_{\mathrm{rel}}$. (Reprinted with permission from reference 6.)

were screened in a search for potential nucleic acid analogues with the necessary characteristics $^{6}$ (Figure 5.1). The pteridines in the lower half of Figure 5.1 were ruled out for further studies since they were not fluorescent enough, not stable, not soluble in aqueous media, or bore no resemblance to native bases.

The pteridines in the top portion of Figure 5.1 are organized according to structural features. Probes 1, 2, and 25 are similar in structure to adenosine while probes 4,5 , and 17 are similar to guanosine. Of the seven that were chosen for further studies, probes 5 and 25 have proven to be quite unstable, while probes 1 and 2 are less favored because of structural disadvantages.

Table 5.1 lists fluorescence properties for the compounds shown in the top portion of Figure 5.1. 3-MI (probe 4) and 6-MI (probe 17) exhibit similar $\mathrm{Q}_{\text {rel }}$ $(0.88-0.70)$ and mean lifetimes $\left(\tau_{\mathrm{m}}\right.$ ranges $\left.5.63-6.54 \mathrm{~ns}\right)$.

At this time two of the four compounds actively being investigated are adenosine analogues, 6MAP and DMAP, which have been developed since the initial screening, and two are guanosine analogues, 3-MI and 6-MI, probes 4 and 
Table 5.1. Fluorescence Properties of Pteridine Nucleoside Analogues ${ }^{a}$

\begin{tabular}{|c|c|c|c|c|c|c|c|c|}
\hline Probe & $\mathrm{Ex}_{\text {max }}$ & $\mathrm{Em}_{\max }$ & $\mathrm{Q}_{\mathrm{rel}}$ & $\tau_{\mathrm{i}}(\mathrm{ns})$ & $\alpha_{i}$ & $\% \mathrm{I}_{\mathrm{i}}$ & $\tau_{\mathrm{m}}$ & $\langle\tau\rangle$ \\
\hline 1 & 354 & 444 & 0.41 & $\begin{array}{l}\tau_{1}=1.91 \\
\tau_{2}=4.05\end{array}$ & $\begin{array}{l}\alpha_{1}=0.19 \\
\alpha_{2}=0.81\end{array}$ & $\begin{array}{l}I_{1}=9.9 \\
I_{2}=90.9\end{array}$ & 3.84 & 3.64 \\
\hline 2 & 358 & 440 & 0.16 & $\begin{array}{l}\tau_{1}=0.76 \\
\tau_{2}=1.05\end{array}$ & $\begin{array}{l}\alpha_{1}=0.67 \\
\alpha_{2}=0.33\end{array}$ & $\begin{array}{l}\mathrm{I}_{1}=59.6 \\
\mathrm{I}_{2}=40.4\end{array}$ & 0.87 & 0.85 \\
\hline 25 & 334 & 443 & 0.27 & $\begin{array}{l}\tau_{1}=2.37 \\
\tau_{2}=4.13\end{array}$ & $\begin{array}{l}\alpha_{1}=0.19 \\
\alpha_{2}=0.81\end{array}$ & $\begin{array}{l}I_{1}=11.6 \\
I_{2}=88.4\end{array}$ & 3.92 & 3.80 \\
\hline 4 & 348 & 430 & 0.88 & $\begin{array}{l}\tau_{1}=3.54 \\
\tau_{2}=6.58\end{array}$ & $\begin{array}{l}\alpha_{1}=0.02 \\
\alpha_{2}=0.98\end{array}$ & $\begin{array}{l}I_{1}=1.2 \\
I_{2}=98.8\end{array}$ & 6.54 & 6.51 \\
\hline 5 & 348 & 430 & 0.87 & $\begin{array}{l}\tau_{1}=1.81 \\
\tau_{2}=6.26\end{array}$ & $\begin{array}{l}\alpha_{1}=0.37 \\
\alpha_{2}=0.63\end{array}$ & $\begin{array}{l}I_{1}=14.6 \\
I_{2}=85.4\end{array}$ & 5.63 & 4.65 \\
\hline 17 & 340 & 431 & 0.70 & $\begin{array}{l}\tau_{1}=5.45 \\
\tau_{2}=6.58\end{array}$ & $\begin{array}{l}\alpha_{1}=0.20 \\
\alpha_{2}=0.80\end{array}$ & $\begin{array}{l}I_{1}=17.5 \\
I_{2}=82.5\end{array}$ & 6.38 & 6.35 \\
\hline 10 & 336 & 400 & 0.54 & $\begin{array}{l}\tau_{1}=3.16 \\
\tau_{2}=8.14\end{array}$ & $\begin{array}{l}\alpha_{1}=0.97 \\
\alpha_{2}=0.03\end{array}$ & $\begin{array}{l}\mathrm{I}_{1}=92.8 \\
\mathrm{I}_{2}=7.2\end{array}$ & 3.52 & 3.31 \\
\hline
\end{tabular}

${ }^{a} \tau_{\mathrm{i}}$, lifetime for each component of a multiexponential model; $\alpha_{\mathrm{i}}$, preexponential for each component of a multiexponential model; $\% \mathrm{I}_{\mathrm{i}}$, percent fluorescence intensity for each component of a multiexponential model; $\langle\tau\rangle$, species-concentration-weighted lifetime; $\tau_{\mathrm{m}}$, intensity-weighted lifetime. (Reprinted with permission from reference 42.$)$

17 of the original set (Figure 5.2). 6MAP and DMAP are similar to probes 1 and 2 except that the phenyl groups are replaced by methyl groups. ${ }^{7}$ The four compounds shown in Figure 5.2 are highly fluorescent, $\mathrm{Q}_{\mathrm{rel}}$ of these ranging from 0.39 to 0.88 . The adenosine analogues, 6MAP and DMAP are at the beginning of their development, but in initial studies they appear be quite similar to the guanosine analogues with respect to stability and quenching effects. $\mathrm{Q}_{\text {rel }}$ for $6 \mathrm{MAP}$ and DMAP are 0.39 and 0.48 , respectively. 6MAP appears to participate in hydrogen bonding with thymidine as evidenced by melting temperatures that are very similar to those of controls. DMAP is currently under investigation.

The overlaid absorption spectra, corrected excitation spectra, and emission spectra of 3-MI and 6-MI are shown in Figure 5.3.

\subsubsection{Effect of $p H$ on Fluorescence Emission}

Emission spectra of 3-MI and 6-MI over a $\mathrm{pH}$ range from 5.0 to 8.0 are shown in Figure 5.4. Varying the $\mathrm{pH}$ had little effect on the emission spectrum of 3-MI, whereas the emission spectrum of 6-MI shifted $10 \mathrm{~nm}$ to the red when the $\mathrm{pH}$ was increased from 7.0 to 8.0.

Similar $\mathrm{pH}$ titrations of DAS on 3-MI and 6-MI were performed. The lifetime components for 3-MI remained unchanged over the $\mathrm{pH}$ range from 7.0 to 9.0 , 


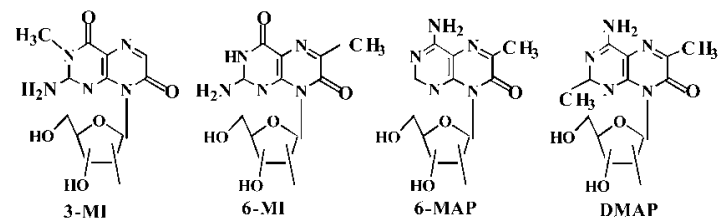

Figure 5.2. Guanosine and adenosine analogues currently under development. (Reprinted with permission from reference 42.)

while with 6-MI there is a blue shift in the longest lived DAS and a smaller increase in the shorter lived component. These DAS demonstrate a pH-dependent equilibrium between a minimum of two emitting species.

\subsubsection{Fluorescence of Pteridine Analogue-Containing Oligonucleotides}

3-MI and 6-MI were incorporated into the oligonucleotide strands shown in Table 5.2 and examined in single-and double-stranded forms (paired to cytidine in the complementary strand). Relative quantum yield $\left(\mathrm{Q}_{\mathrm{rel}}\right)$ measurements of 3-MI-containing oligonucleotides (21-mers) in which the fluorophore is substituted for guanosine at several positions are also listed in Table 5.2.

In PTER8 the fluorophores are incorporated into a purine-rich segment of the oligonucleotide (Tables 5.2 and 5.3) and fluorescence of both probes is substantially quenched, whereas in PTER9 the fluorophores are surrounded by pyrimidines resulting in less quenching of the fluorescence signal. These were found to be the two extreme conditions for neighboring base quenching of the fluorophores.

With 3-MI, double strand formation led to less additional quench over that seen in the single-stranded oligonucleotide. In the PTER9 oligonucleotide, degree of quench relative to unincorporated 3-MI was $64 \%$ and $68 \%$ in the single- and double-stranded forms, respectively. For 6-MI, the degree of quench in PTER9 increased from $56 \%$ to $64 \%$ on going from the single- to the double-stranded form. The emission spectrum of 6-MI shifts $7 \mathrm{~nm}$ to the red when it is incorporated into a single- or double-stranded oligonucleotide compared to its unincorporated monomer form. The emission spectrum of 3-MI shifts only $2 \mathrm{~nm}$ when incorporated into an oligonucleotide.

The lifetimes, mean lifetimes, and amplitudes for 3-MI and 6-MI incorporated into single-stranded and double-stranded oligonucleotides are also listed in Table 5.3. The dominant component of the intensity decay curves becomes more complex and components of the decay curve with shorter lifetimes becomes more prominent. For example, 3-MI in PTER8 has a tri-exponential decay curve with the predominant component having a lifetime of $0.21 \mathrm{~ns}$. 

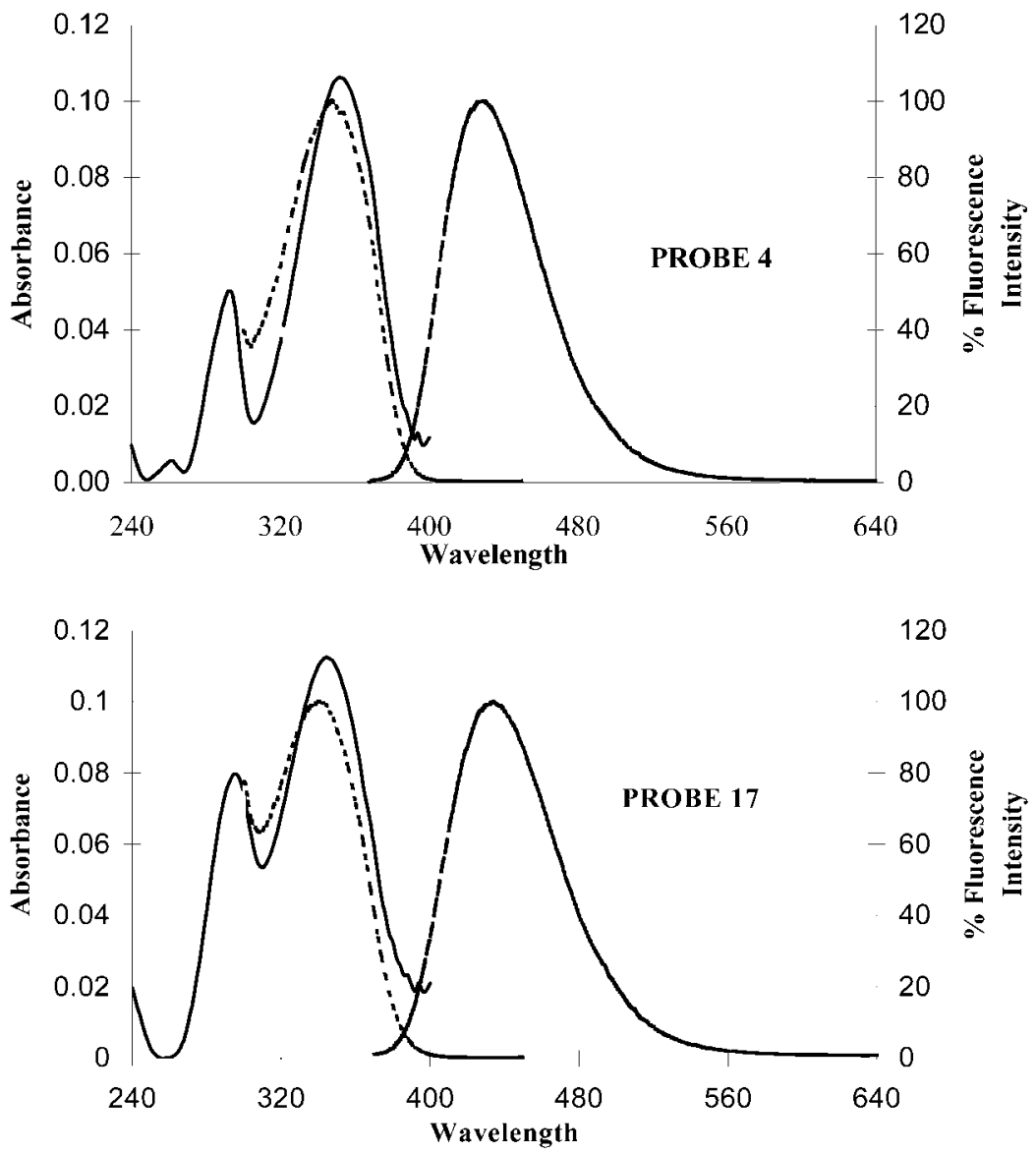

Figure 5.3. Overlay of absorption spectra, corrected excitation spectra, and emission spectra for probes 4 (3-MI) and 17 (6-MI). Excitation and emission are expressed as a percent of maximum. Solid line is absorption, dashed line is excitation, and dotted line is emission. (Adapted with permission from reference 6.)

\subsubsection{Melting Temperatures}

Melting temperatures $\left(\mathrm{T}_{\mathrm{m}}\right)$ were measured on a series of probe-containing oligonucleotides as well as on the identical strands containing no probe and strands containing a single-base pair mismatch at the identical position to the fluorophore (Table 5.4). For 3-MI-containing oligonucleotides, Tm depression was approximately equivalent to a single base-pair mismatch in the same position.

These probes are quite stable and tolerate normal handling through synthesis 

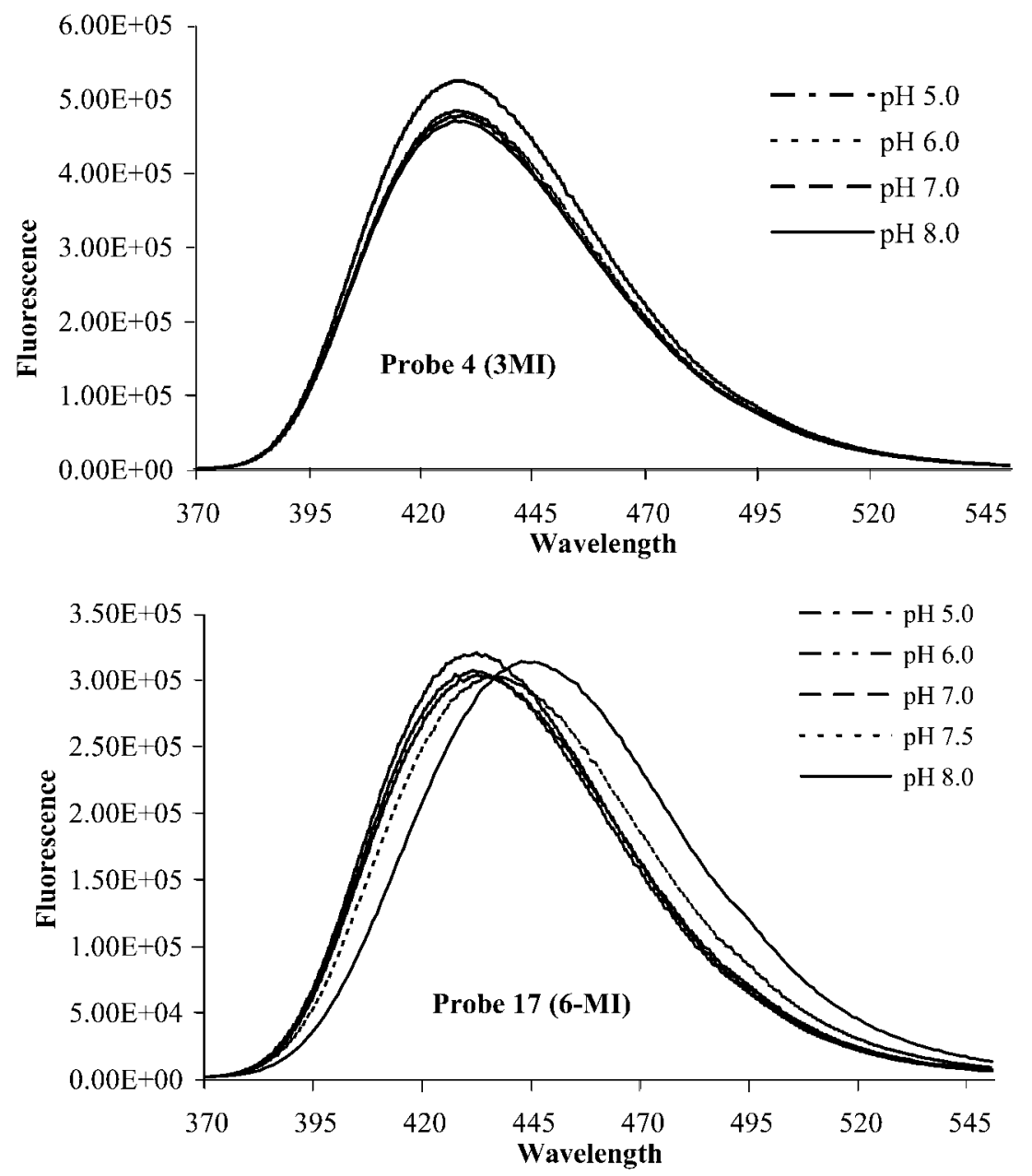

Figure 5.4. Fluorescence emission spectra of Probe 4 (3-MI) and Probe 17 (6-MI) at pH 5.0 to 8.0. (Adapted with permission from reference 6.)

as phosphoramidites and incorporation into oligonucleotides through the normal phosphodiester linkage using a standard DNA synthesizer. 3-MI requires no variations from standard de-blocking methods. 6-MI must be manually deblocked using a $1 \mathrm{M}$ solution of 1,8-diazabicyclo[5.4.0] undec-7-ene (DBU) while still attached to the column. Details are available on the website for TriLink BioTechnologies, Inc. at www.trilinkbiotech.com or through the author. Typically, 
Table 5.2. Oligonucleotides Containing 3-MIa

\begin{tabular}{|c|c|c|}
\hline Name & Sequence & Quantum yield \\
\hline PTER1 & 5'-GTF TGG AAA ATC TCT AGC AGT-3' & 0.13 \\
\hline PTER2 & 5'-GTG TFG AAA ATC TCT AGC AGT-3' & 0.10 \\
\hline PTER3 & 5'-GTG TGF AAA ATC TCT AGC AGT-3' & 0.03 \\
\hline PTER4 & 5'-GTG TGG AAA ATC TCT AFC AGT-3' & 0.06 \\
\hline PTER5 & 5'-GTG TGG AAA ATC TCT AGC AFT-3' & 0.14 \\
\hline PTER7 & 5'-ACT GCT AGA FAT TTT CCA CAC-3' & 0.04 \\
\hline PTER8 & 5'-ACT GCT AFA GAT TTT CCA CAC-3' & 0.05 \\
\hline PTER9 & 5'-ACT FCT AGA GAT TTT CCA CAC-3' & 0.29 \\
\hline
\end{tabular}

${ }^{a}$ Position of the fluorophore is denoted by the $\mathbf{F}$. The relative quantum yield for those containing 3-MI are listed at right. (Reprinted with permission from reference 42.)

Table 5.3. Fluorescence Properties of Probe-Containing Oligonucleotides in Single and Double Strands ${ }^{a}$

\begin{tabular}{lccccccc}
\hline Probe & Pter & & Q\% & $\tau_{\mathrm{i}}$ & $\alpha_{\mathrm{i}}$ & $\%_{\mathrm{i}}$ & $\tau_{\mathrm{m}}$ \\
\hline 3-MI & \multirow{2}{*}{8} & ss & 96 & $\tau_{1}=2.35$ & $\alpha_{1}=0.41$ & $\mathrm{I}_{1}=21.2$ & 5.27 \\
& & & & $\tau_{2}=6.06$ & $\alpha_{2}=0.59$ & $\mathrm{I}_{2}=78.8$ & \\
3-MI & 8 & ds & 96 & $\tau_{1}=0.21$ & $\alpha_{1}=0.70$ & $\mathrm{I}_{1}=10.0$ & 4.60 \\
& & & & $\tau_{2}=2.88$ & $\alpha_{2}=0.16$ & $\mathrm{I}_{2}=32.5$ & \\
& & & & $\tau_{3}=6.35$ & $\alpha_{3}=0.13$ & $\mathrm{I}_{3}=57.5$ & \\
3-MI & 9 & ss & 64 & $\tau_{1}=2.54$ & $\alpha_{1}=0.31$ & $\mathrm{I}_{1}=17.8$ & 4.74 \\
& & & & $\tau_{2}=5.22$ & $\alpha_{2}=0.69$ & $\mathrm{I}_{2}=82.2$ & \\
3-MI & 9 & ds & 68 & $\tau_{1}=1.86$ & $\alpha_{1}=0.33$ & $\mathrm{I}_{1}=14.5$ & 4.81 \\
& & & & $\tau_{2}=5.31$ & $\alpha_{2}=0.67$ & $\mathrm{I}_{2}=85.4$ & \\
6-MI & 8 & ss & 96 & $\tau_{1}=0.30$ & $\alpha_{1}=0.81$ & $\mathrm{I}_{1}=25.6$ & 3.90 \\
& & & & $\tau_{2}=2.21$ & $\alpha_{2}=0.14$ & $\mathrm{I}_{2}=31.3$ & \\
& & & & $\tau_{3}=7.28$ & $\alpha_{3}=0.06$ & $\mathrm{I}_{3}=43.1$ & \\
6-MI & 8 & ds & - & $\tau_{1}=0.21$ & $\alpha_{1}=0.83$ & $\mathrm{I}_{1}=33.0$ & 2.59 \\
& & & & $\tau_{2}=1.20$ & $\alpha_{2}=0.13$ & $\mathrm{I}_{2}=30.4$ & \\
& & & & $\tau_{3}=5.89$ & $\alpha_{3}=0.03$ & $\mathrm{I}_{3}=36.6$ & \\
6-MI & 9 & ss & 56 & $\tau_{1}=3.45$ & $\alpha_{1}=0.25$ & $\mathrm{I}_{1}=14.1$ & 6.51 \\
& & & & $\tau_{2}=7.01$ & $\alpha_{2}=0.75$ & $\mathrm{I}_{2}=85.9$ & \\
6-MI & 9 & ds & 64 & $\tau_{1}=0.26$ & $\alpha_{1}=0.88$ & $\mathrm{I}_{1}=43.4$ & 2.79 \\
& & & & $\tau_{2}=1.04$ & $\alpha_{2}=0.09$ & $\mathrm{I}_{2}=17.0$ & \\
& & & & $\tau_{3}=6.30$ & $\alpha_{3}=0.03$ & $\mathrm{I}_{3}=39.6$ & \\
\hline
\end{tabular}

${ }^{a}$ Measurements were taken in $10 \mathrm{mM}$ Tris buffer, $\mathrm{pH} 7.5$ at room temperature. See Table 5.2 for oligonucleotide sequence and position of fluorophore within the strand. Studies were performed on single-stranded fluorophore-containing oligonucleotide (single) and on the identical oligonucleotide annealed to its complementary strand (double). The degree of quench of the fluorescence emission is relative to the deoxyribo-pteridine nucleoside analogue (monomer) Q\%. Quantum yields of probes 4 (3-MI) and 17 (6-MI) are listed in Table 5.2. Abbreviations: $\tau_{i}$, lifetime for each component of a multiexponential model; $\alpha_{i}$, preexponential for each component of a multiexponential model; $\% \mathrm{I}_{\mathrm{i}}$, percent fluorescence intensity for each component of a multiexponential model; $\tau_{\mathrm{m}}$, intensity-weighted lifetime. (Reprinted with permission from reference 42.) 
Table 5.4. Effects of the Position of the Fluorophore on the Melting Temperatures for 3-MI and 6-Mla

\begin{tabular}{llll}
\hline Oligonucleotide & 3-MI & 6-MI & Mismatch \\
\hline PTER1 & $56.0^{\circ} \mathrm{C}$ & & \\
PTER2 & $55.6^{\circ} \mathrm{C}$ & & \\
PTER3 & $53.8^{\circ} \mathrm{C}$ & $63.6^{\circ} \mathrm{C}$ & $54.4^{\circ} \mathrm{C}$ \\
PTER4 & $52.0^{\circ} \mathrm{C}$ & & $54.4^{\circ} \mathrm{C}$ \\
PTER5 & $58.8^{\circ} \mathrm{C}$ & $62.6^{\circ} \mathrm{C}$ & \\
PTER7 & $58.4^{\circ} \mathrm{C}$ & & \\
PTER8 & $50.4^{\circ} \mathrm{C}$ & $61.6^{\circ} \mathrm{C}$ & \\
PTER9 & $54.6^{\circ} \mathrm{C}$ & & \\
\hline
\end{tabular}

${ }^{a}$ The site of fluorophore incorporation is shown in Table 5.2. $\mathrm{T}_{\mathrm{m}} \mathrm{s}$ were measured in $10 \mathrm{mM}$ Tris, $\mathrm{pH} 7.5$ with $10 \mathrm{mM}$ $\mathrm{NaCl}$. The $\mathrm{T}_{\mathrm{m}}$ of the control double strand was $63.2^{\circ} \mathrm{C}$. (Reprinted with permission from reference 6.)

probe-containing oligonucleotides are purified through polyacrylamide gel electrophoresis and ethanol precipitation. Although incorporation into DNA significantly quenches the fluorescence signal, the selected fluorophores start with quantum yields as high as 0.88 and can still be easily detected within oligonucleotides. Changes in the local structure of fluorophore-containing oligonucleotides can be monitored by measuring changes in fluorescence properties.

The significant quenching seen upon incorporation of 3-MI and 6-MI into single-stranded oligonucleotides is related to the proximity of purines to the fluorophore in the strand. The majority of quench is observed upon incorporation into the oligonucleotide with a less severe additional quench upon annealing the fluorophore-containing oligonucleotide to its complementary strand, suggesting that quenching originates mostly in base stacking interactions rather than base pairing. For PTER8, containing the fluorophore surrounded by purines, the fluorescence signal is almost completely quenched $(96 \%)$ in the single-stranded form, and little additional quench is detected in the double-stranded form. For PTER9 containing 6-MI, quench increases from 56\% to $64 \%$ with annealing, and for PTER9 containing 3-MI there is a smaller increase in quench from $64 \%$ to $68 \%$ when the labeled strand is annealed to its complement.

The $\mathrm{T}_{\mathrm{m}}$ studies suggest that 3-MI does not participate fully in base pairing in double-stranded oligonucleotides because the $\mathrm{T}_{\mathrm{m}}$ depression in 3-MI-containing oligonucleotides is approximately equivalent to that of a single base pair mismatch. In contrast, the $\mathrm{T}_{\mathrm{m}} \mathrm{s}$ of double-stranded 6-MI -containing oligonucleotides are very similar to the $\mathrm{T}_{\mathrm{m}} \mathrm{s}$ of the controls, suggesting that 6-MI may participate in base pairing. The shift in emission spectra of 6-MI going from single-stranded to 
double-stranded form is also consistent with base pairing. A shift of this type was not observed for double-stranded 3-MI (Figure 5.4).

The effects of quenchers or energy transfer interactions may also be monitored through lifetimes. For example, if a fluorophore within an oligonucleotide is shielded because of an interaction between the oligonucleotide and a protein, the lifetime will not be affected by the introduction of extrinsic dynamic quenchers (e.g., $\mathrm{Cs}^{+}$or $\mathrm{I}^{-}$). However, if the probe is in a more exposed position within the oligonucleotide, then the lifetime will be affected by the addition of dynamic quenchers. Similarly, a probe may be dynamically quenched by collisions with intrinsic quenchers, e.g., neighboring groups such as carbonyl oxygen.

In its monomer form, 3-MI has one major lifetime component of $6.58 \mathrm{~ns}$ $(99 \%)$ and a $\langle\tau\rangle$ of $6.51 \mathrm{~ns}$. Upon incorporation into PTER8, the decay curve becomes more complex and two distinct lifetimes of $2.35 \mathrm{~ns}(41 \%)$ and $6.06 \mathrm{~ns}$ (59\%) are required to describe the decay curve, resulting in a $\langle\tau\rangle$ of 4.54 ns. Annealing this fluorophore-containing oligonucleotide to its complementary strand forming the double-stranded PTER8 increases the complexity even further, requiring a third, dominant short-lived component $(0.21 \mathrm{~ns})$ to fit the decay curve (Table 5.3), and the $\langle\tau\rangle$ drops to $1.45 \mathrm{~ns}$. This latter change may provide a convenient means of monitoring whether an oligonucleotide (or a segment of an oligonucleotide) containing one of the fluorophores is in the single- or doublestranded state. In PTER9 the degree of quench associated with incorporation of 3-MI into the oligonucleotide is less, and there is no increase in the complexity of the decay curve and no substantial change in $\langle\tau\rangle$ in the double strand.

For 6-MI, incorporation into PTER8 is also associated with substantial quenching, an increase in the complexity of the decay curve from two to three components, and a much shorter $\langle\tau\rangle(6.35 \mathrm{~ns}$ for the monomer and $0.95 \mathrm{~ns}$ in the single-stranded oligonucleotide). The double-stranded, 6-MI-containing PTER8 oligonucleotide has a slightly shorter $\langle\tau\rangle(0.53 \mathrm{~ns})$ than the single-stranded oligonucleotide. The change in the shape of 6-MI's decay curve and the decrease in $\langle\tau\rangle$ resulting from annealing the fluorophore-containing single-stranded PTER9 to its complementary strand is more dramatic (Table 5.3). Based on the Tm measurements, 6-MI appears to participate in base-pairing interactions, while 3-MI does not. This may in part explain the differences in the observed changes in fluorescence properties of 3-MI- and 6-MI-containing oligonucleotides.

Comparing changes in $\langle\tau\rangle$ and relative quantum yield (expressed as percentage quench in Table 5.3) in the monomer and the fluorophore-containing singleand double-stranded oligonucleotide provides insight into the mechanism of the quench resulting from incorporation of the pteridine nucleoside into an oligonucleotide. Static quench should not be accompanied by a change in $\langle\tau\rangle$, while pure dynamic quench is associated with proportional changes in quantum yield and $\langle\tau\rangle$. Disproportionate changes in quantum yield and $\langle\tau\rangle$ suggest that quenching is due to a combination of static and dynamic events. The tertiary structure of the oligonucleotide could expose the fluorophore to collisional events from its sur- 
roundings (dynamic quenching) or could position the fluorophore in contact with other bases or backbone quenchers within the oligonucleotide (static quenching). In the 3-MI-containing PTER8 single-stranded oligonucleotide, the fluorophore is surrounded by purine bases and the $\mathrm{Q}_{\text {rel }}$ is $96 \%$ quenched compared with the monomer. The $\langle\tau\rangle$ of 3-MI is also shorter in the single-stranded oligonucleotide, but the change in $\langle\tau\rangle$ is only $30 \%$ (6.51 to $4.54 \mathrm{~ns}$ ), suggesting that static quench arising from the surrounding purines is the primary mechanism involved in the quenching of the fluorescence signal. A more detailed analysis and discussion of the quenching mechanisms of 3-MI containing single and double strands has been done by Driscoll et al. ${ }^{8}$

The $\mathrm{pH}$ titration of the emission spectra and DAS of 3-MI and 6-MI yielded a shift in the emission spectrum of 6-MI between pHs of 7.0 to 8.0, and an increase in one of 6-MI's lifetime components over the $\mathrm{pH}$ range from 7.0 to 9.0. These DAS also provide a clear signature of an excited state reaction. ${ }^{9}$ This property of 6-MI could be exploited to measure local $\mathrm{pH}$ and buffering. The shift in the emission spectrum of 6-MI was not seen with 3-MI and suggests that there is a protonation at the 3-position, which is protected by the methyl group in 3-MI. A minimum of two emitting species (e.g., tautomers) would be needed to explain these DAS.

In the following sections, we will demonstrate a number of applications that utilize these highly fluorescent analogues in ways that take advantage of their unique properties.

\subsection{Applications Using Pteridines}

\subsubsection{Integrase Assay}

One way to use these analogues is to simply monitor changes in fluorescence intensity when some action physically removes them from base-stacking interactions. The following example demonstrates this using the endonucleolytic cleavage activity of a protein from HIV $-1{ }^{1}$

Integrase is a retrovirally-encoded protein responsible for the integration of viral DNA into the host cell's genome, a critical step in the life cycle of a retrovirus. This protein functions in a step-wise manner, the first step being cleavage of a dinucleotide from both of the $3^{\prime}$-ends ( $3^{\prime}$-processing reaction). Cleavage is followed by other steps, resulting in the integration of the HIV-1 genome into the host cell's DNA. The fluorescent pteridine nucleoside analogue based assay focuses on the cleavage step of this reaction.

The integrase catalyzed 3 '-processing (endonucleolytic cleavage) and strand transfer reactions have previously been studied in vitro using recombinant protein and short double-stranded oligonucleotides (21-mers) with sequences identical to 
either the U5 or U3 terminus of HIV-1 DNA as substrates. ${ }^{10-13}$ The substrate is ${ }^{32} \mathrm{P}-$ labeled and the reaction products are analyzed by polyacrylamide gel electrophoresis and autoradiography to detect cleavage (19-mer) or strand transfer ( 30mer) products.

In the fluorescence-based assay utilizing the pteridine nucleoside analogue, 3-MI, the probe is site-specifically inserted into the cleavage site of a doublestranded oligonucleotide substrate. Cleavage of the $3^{\prime}$-terminal dinucleotide containing 3-MI results in a measurable real time increase in fluorescence intensity. Figure 5.5 shows a schematic of the integrase cleavage step with release of 3-MI (F) from the oligonucleotide.

Fluorescence intensity changes resulting from the integrase 3 '-processing reaction are depicted in Figure 5.6. This assay is based on an in vitro cell-free and virus-free system in which purified recombinant HIV-1 integrase protein is incubated with a short duplex oligonucleotide (21-mer), with a sequence identical to the U5 terminus of viral DNA. ${ }^{10-12,14} 3-\mathrm{MI}$ is substituted for guanosine at the cleavage site of the oligonuceotide substrate. As part of the 3' processing reaction, the terminal 3' -dinucleotide, containing 3-MI, is cleaved from the oligonucleotide and the quenching effect of the neighboring adenosine is abrogated resulting in a marked increase in the fluorescence intensity of the reaction mixture.

The increase in fluorescence intensity was linear for up to $20 \mathrm{~min}$. The rate of the increase in fluorescence intensity is proportional to the rate of the integrase 3 '-processing reaction and, because the change in fluorescence intensity is dependent on release of the 3-MI-containing dinucleotide, this assay is specific for the integrase 3 '-processing reaction. The potential increase in fluorescence intensity from the release of the 3-MI-containing dinucleotide is also illustrated by the marked difference in the fluorescence emission profiles of the 3-MI-containing oligonucleotide and the 3-MI-containing dinucleotide shown in Figure 5.7.

\section{GTGTGGAAAATCTCTAGCAFT - 3' CACACCTTTTAGAGATCGTCA - 5'}

Integrase

GTGTGGAAAATCTCTAGCA $-3^{\prime}+\mathbf{F T}-3^{\prime}$ CACACCTTTTAGAGATCGTCA - 5'

Figure 5.5. Integrase 3 '-processing reaction with 3-MI-containing substrate. The cleavage reaction of integrase releases the 3-MI containing $3^{\prime}$-terminal dinucleotide from the strand. 3-MI is denoted as $\mathbf{F}$. (Reprinted with permission from reference 42.) 


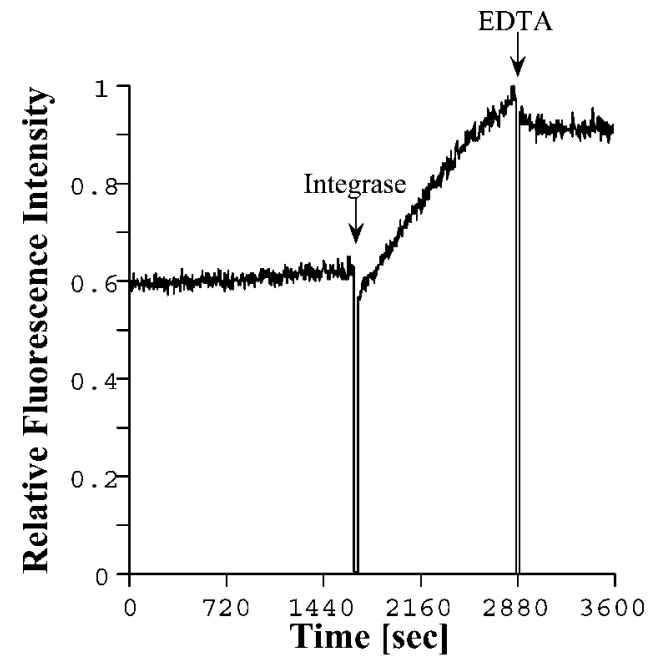

Figure 5.6. Change in fluorescence intensity over time during incubation of HIV-1 integrase with the 3-MI-containing oligonucleotide substrate. The real time kinetic trace of fluorescence intensity depicts the blank rate prior to addition of integrase to the reaction mixture ( 0 to $1700 \mathrm{~s})$, the increase in fluorescence intensity following addition of integrase to the reaction mixture (1700 to $2880 \mathrm{~s}$ ), and cessation of the reaction following the addition of EDTA ( $>2880 \mathrm{~s}$ ) which chelates manganese, a required cofactor. The fluorescence emission was measured at $400 \mathrm{~nm}$ following the excitation at 360 $\mathrm{nm}$. (Reprinted with permission from reference 1).

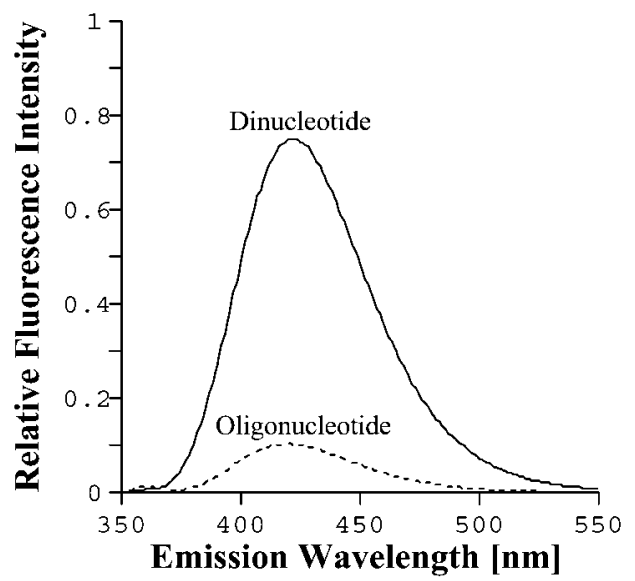

Figure 5.7. Comparison of the fluorescence emission spectra for the 3-MI-containing substrate oligonucleotide and the 3-MI-containing dinucleotide cleavage product. Excitation was at $360 \mathrm{~nm}$. The oligonucleotide and the dinucleotide solutions were analyzed at equimolar concentrations as measured by absorbance of the solution at $360 \mathrm{~nm}$. (Reprinted with permission from reference 1.) 
The removal of the fluorophore from a single- or double-stranded oligonucleotide through the action of any endo- or exonuclease may be presumed to be easily monitored through changes in fluorescence intensity as well. This increase has also been demonstrated using other enzymes, including P1 nuclease and Exonuclease III.

\subsubsection{Bulge Hybridization Probes}

The unique properties of the pteridine nucleoside analogues provide us with a very convenient method for detection of hybridization of oligonucleotides. Since the quench in fluorescence intensity is largely attributable to base stacking interactions, it was assumed that any disruption in the base stacking arrangement would have a direct influence on the fluorescence intensity. Disruption in the helical arrangement of bases in an oligonucleotide can be caused by various means. This application explores the effects of forcing a one, two, or three base bulge within a double strand.

The application of the fluorophores as bulge hybridization probes utilizes the probe incorporated into an oligonucleotide that is perfectly complementary to a known target sequence, except for the addition of the fluorophore. When annealing occurs, the probe is squeezed out of the strand since it does not have a basepairing partner. Since this partially relieves fluorescence quenching induced by interactions with neighboring bases, a direct increase in fluorescence intensity is observed (Figure 5.8). This technique is used to determine the presence or absence of specific DNA sequences in a mixture without the need for separation of annealed or labeled products. Fluorescence increases upon bulge formation range from -1.5 -fold to 27 -fold depending on the sequence. Although some increase in fluorescence intensity is realized with 2 or 3 base bulges, the highest signal increase is seen with the single base bulge.

The most productive bulge occur within a group of adenosines. Since this is also the most quenched environment for 3-MI, it provides a very low background.

The degree of increase revealed by annealing the two strands is dependent on the identity of the bases nearest to the fluorophore. The combination of selectivity and the fact that the products do not need to be separated for analysis make this a very rapid and attractive technique.

This bulge hybridization technique has been used to measure the products of polymerase chain reactions (PCR) without the need for separation techniques (Hawkins et al., unpublished). In this approach, we synthesized a probecontaining strand that was complementary to a section of the region of product between (but not overlapping) the primers. An investigation of the advantages and disadvantages of using multiple probes per strand revealed that each 3-MI bulge depresses the Tm of the double strand by $1-2^{\circ} \mathrm{C}$. We designed a PCR probe strand 


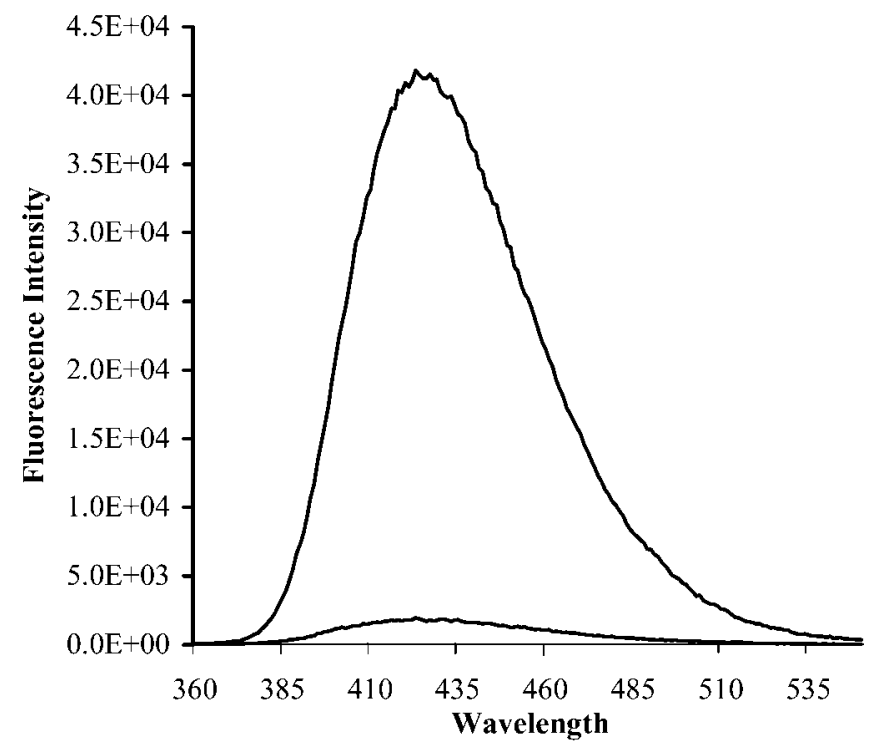

Figure 5.8. Difference in fluorescence intensity resulting from hairpin formation as measured by integration of the area under each peak. The two 3-MI-containing strands are equimolar. Hybridization was done by heating the strands to $95^{\circ} \mathrm{C}$ for two minutes and allowing them to cool to room temperature. Excitation was at $350 \mathrm{~nm}$. The larger peak is the scan of the double-stranded material of the sequence 5 '-agcagttgctaaagaaFaattgaacacgctcggacttgc- $3{ }^{\prime}$. This is measured to be a 21 -fold increase.

containing two 3-MI molecules designed to form bulges when annealing occurs. The probe strand is added to the PCR mixture prior to amplification at which time no complementary strands are present. As new product is created in the process of the amplification, complementary strands (targets) become available for the probe to bind resulting in increasing fluorescence intensity. Products containing no template (blank) and a series of known template concentrations were scanned at the conclusion of 30 cycle. Positive PCR reactions exhibit up to threefold increase in fluorescence intensity over the blank and showed a linear correlation between the amount of template and the amount of fluorescence increase. Results were confirmed using standard agarose gel electrophoresis.

\subsubsection{Anisotropy}

Studies done at Wesleyan University by Mukerji et al. ${ }^{15}$ have utilized steady state fluorescence anisotropy using 3-MI fluorescence to monitor the binding of the HU protein to duplex DNA. HU, a multifunctional histone-like protein found 
only in prokaryotes, does not recognize or bind to a particular sequence of DNA, but does bind preferentially to supercoiled and bent DNA. This protein contains no Trp or Tyr residues; therefore, to probe the binding interaction by fluorescence spectroscopy it was necessary to label the DNA duplexes with 3-MI. This probe was specifically incorporated at one site into a 13-bp and a 34-bp DNA duplex. The binding affinity of the protein as well as the conformation of DNA were addressed in these experiments.

Anisotropy measurements were performed at a constant concentration of DNA with increasing concentrations of HU protein. Under these conditions the binding curves (Figure 5.9) yielded a Kd value of $1.51 \times 10^{-6} \mathrm{M}$ for the 34-bp duplex (3MI-34) and a value of $3.16 \times 10^{-6} \mathrm{M}$ for the 13-bp duplex (3MI-13). As shown by the fits, the interaction is well described by a binding equation that assumes a 1:1 interaction in both cases. The observed difference in binding constants for the two duplexes is larger than the error associated with the fit and is suggestive that the flanking regions are relevant for the interaction, since binding to $3 \mathrm{MI}-34$ is slightly stronger than binding to $3 \mathrm{MI}-13$. Previous studies ${ }^{16}$ had suggested that HU binds to 9 bp of DNA; however, fluorescence anisotropy measurements are more consistent with a 1:1 interaction for both the 13- and 34-bp duplexes. Interestingly, in gel mobility shift assays (GMSA) performed with

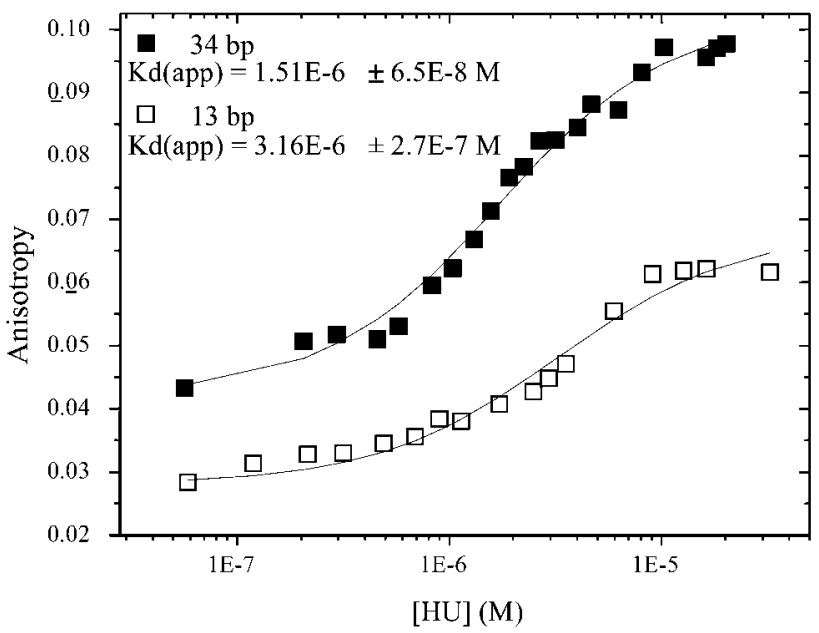

Figure 5.9. The binding of HU to $13 \mathrm{bp}$ and $34 \mathrm{bp}$ duplex oligomers as monitored by fluorescence anisotropy is shown. Excitation wavelength was $330 \mathrm{~nm}$ and anisotropy was monitored from 409 to $417 \mathrm{~nm}$. Plotted values are an average with an error of \pm 0.0025 . Samples were in a $15 \mathrm{mM} \mathrm{KCl}, 2 \mathrm{mM}$ spermidine, $0.1 \mathrm{mM}$ EDTA, $10 \mathrm{mM}$ Tris $\mathrm{pH}=7.6$ buffer. Temperature was maintained at $4^{\circ} \mathrm{C} . \mathrm{Kd}(\mathrm{app})$ values were determined by assuming a 1:1 binding interaction with the fits as shown. 
3MI-34, formation of 1:1 and higher order HU:DNA complexes $(2: 1,3: 1,4: 1$, etc.) was observed. In this case the $\mathrm{Kd}(\mathrm{app})$ determined by GMSA is $5.0 \times 10^{-8} \mathrm{M}$, which is significantly smaller ( 30-fold) than the value determined by anisotropy. The observation of multiple HU:DNA complexes by GMSA has been previously reported with other DNA duplexes of comparable length. ${ }^{17}$ For 3MI-13, however, only one complex is observed in the GMSA, and the determined $\operatorname{Kd}(\mathrm{app}), 1.30 \times$ $10^{-6} \mathrm{M}$, is similar to that determined by fluorescence anisotropy (see above). Despite the differences in the absolute determination of the $\operatorname{Kd}(\mathrm{app})$, both the GMSA and fluorescence measurements are suggestive that HU has a higher binding affinity for 3MI-34 relative to 3MI-13.

Fluorescence stoichiometry experiments performed with 3MI-34 are consistent with the anisotropy experiments and are indicative of a binding stoichiometry of at most 2:1 HU:DNA. Thus, we suggest that the numerous complexes observed in the GMSA result not from higher order protein:DNA complexes but altered migration patterns as a consequence of DNA bending and the geometry of the bend. HU has striking primary and secondary structural similarities with a homologous protein, integration host factor (IHF), and the mode of interaction with DNA may be similar to that of IHF. IHF forms a 1:1 complex with DNA and protein binding induces the DNA to form a bend of $\sim 160 .{ }^{18}$ Similarly, HU exhibits a marked preference for bent DNA and facilitates circularization of DNA. ${ }^{19}$ Upon binding to $\mathrm{HU}, 3 \mathrm{MI}-34$ exhibits an increase in fluorescence intensity, which is consistent with increased solvent exposure of the nucleoside analogue. This increase in fluorescence intensity is strongly suggestive of a structural deformation of the DNA that leads to an unwinding of the helix and possible unstacking of the DNA bases. This result is also supported by the observed change in anisotropy upon protein binding (see below). No fluorescence intensity change is observed for 3MI-13 upon binding to $\mathrm{HU}$, which is indicative of 3-MI remaining in a stacked configuration. We suggest that the relatively short length of this DNA is prohibitive for bending. The absence of a fluorescence intensity change is consistent with the observation of only one band in the DNA binding gel and is suggestive of little or no change in the conformation of $3 \mathrm{MI}-13$ upon binding to HU.

The anisotropy of $3 \mathrm{MI}-13$ alone is 0.02 compared to a value of 0.04 for $3 \mathrm{MI}-34$; thus, the $r(0)$ values reflect the length difference of the two duplexes. The overall anisotropy change upon protein binding is similar for the two duplexes, but is more pronounced in the case of 3 MI-34 (0.054 vs. 0.039). This increment, while larger, may in fact be reduced by an increase in lifetime upon binding. The relationship between the correlation time $(\phi)$ and lifetime $(\tau)$ is shown in a form of the Perrin equation shown below.

$$
\bar{r}=\frac{\bar{r}_{0}}{1+\bar{\tau} / \bar{\phi}}
$$


Consequently, this contrast probably would be greater if changes in had been measured instead of changes in anisotropy. If both measurements monitor the binding of $1 \mathrm{HU}$ dimer to DNA, as suggested by the stoichiometry experiments and the analysis of the binding curve, the bound correlation time change should be the same. The larger anisotropy change for 3MI-34 potentially results from a change in conformation of the DNA upon binding. This is supported by the concomitant increases in fluorescence intensity and anisotropy. The intensity increase is suggestive of local unstacking of the DNA bases or unwinding of the helix, since the quantum yield of 3-MI is larger in single-stranded DNA relative to double-stranded. Thus, in contrast to the GMSA, fluorescence experiments (intensity, anisotropy, and stoichiometry) are more consistent with a 1:1 HU:DNA inteaction and are also strongly indicative of a change in DNA conformation upon binding, such that in the $34 \mathrm{bp}$ duplex 3-MI becomes solvent exposed. The absence of a fluorescence intensity increase upon HU binding to $3 \mathrm{MI}-13$ is suggestive that it is too short to experience a similar change in conformation. These fluorescence binding experiments provide relevant information regarding the protein-DNA interaction especially with respect to DNA conformation, and are suggestive of a new interpretation of the GMSA data.

\subsubsection{Intracellular Transport of Oligonucleotides}

Studies are being carried out at Mt. Sinai in New York by Basil Hanss et al. (unpublished) using 3-MI as a probe for oligonucleotide transport. Cellular uptake of oligonucleotides has important therapeutic implications. Genes can be transferred to cells using plasmid DNA, protein synthesis can be interrupted with ribozymes or antisense oligodeoxynucleotides, and, in emerging technology, genetic defects caused by point mutations can be corrected with RNA/DNA hybrid oligonucleotides. ${ }^{20}$ All of these promising approaches require internalization of oligonucleotides (ODNs) at appropriate intracellular locations. Overall oligonucleotide uptake by most tissues in vivo is relatively low, the highest being in kidney, liver, and brain. ${ }^{21-25}$ The aim of this study is to define the molecular mechanism of oligonucleotide internalization.

The molecular mechanism of ODN uptake is not completely understood, although it is known that uptake is saturable and energy requiring in most tissues. ${ }^{25-29}$ A considerable body of literature exists describing the kinetics of ODN transport and, based on those studies, three transport mechanisms have been proposed: receptor mediated endocytosis, fluid phase endocytosis (i.e., pinocytosis), and transport through an oligonucleotide conducting channel.

Kinetics studies, for the most part, have used ODNs labeled with either a 
radioisotope or a fluorescent tag, most commonly end labeling with FITC or a similar fluorophore. Evidence in the literature reports different rates of uptake for radio-labeled and FITC-labeled ODNs. ${ }^{30}$ In order to further explore the source of this difference, 3-MI will be incorporated into an oligonucleotide.

Three general areas of investigation will be optimized for use with 3-MI. ${ }^{1}$ Oligonucleotides containing 3-MI (3MI-ODN) to measure ODN uptake/efflux kinetics. An extremely bright oligonucleotide containing six 3-MI molecules in a $22-$ mer is being used to increase sensitivity $\left(5^{\prime}-\mathrm{Ftc} c \mathrm{Fc}\right.$ tcF tct Fct cFt tcF c-3'). Currently, radiolabeled ODNs are being used to study uptake kinetics, transepithelial flux, and the molecular mechanism of ODN transport across both the apical and basolateral membranes of renal epithelia. The model systems consist of primary cultures of human renal tubule cells and isolated perfused renal tubules. Addition of 3MI-ODN into these studies will allow real time measurement of ODN flux using confocal fluorescence microscopy. ${ }^{2}$ The intracellular fate of ODNs in renal tubule cells is unclear; yet their therapeutic efficacy is absolutely dependent upon trafficking to the correct intracellular compartment. Use of 3MI to follow trafficking will provide a more native-like model than that previously used. ${ }^{3}$ Finally, to be effective for antisense purposes, ODNs must hybridize to target mRNAs; however, little is known about the in vivo binding of ODNs to target sequences and it is difficult to predict which sequences will bind efficiently to the target mRNA. Pteridine nucleoside analogue-containing ODNs are being used to develop an in vivo hybridization assay to address these important issues.

\subsubsection{DNA Conformation}

Laws and others at Mt. Sinai in New York, ${ }^{7}$ have studied nucleoside analogues using time-resolved fluorescence resonance energy transfer. Studies done by Parkhurst et al. ${ }^{31-33}$ have greatly enhanced our understanding of DNA conformation and dynamics. Typically, resonance energy transfer data provides information about the distribution of distances between donor and acceptor fluorophores. If the donor and acceptor are attached to specific sites on a macromolecule, this distance distribution can provide invaluable insights into structure and conformational dynamics. Since nucleoside analogues exist in a very restricted conformation within the DNA, the donor-acceptor distance distributions experienced by the nucleoside analogue will arise predominantly from the conformation and dynamics of the DNA itself. In the studies by Parkhurst, measurements reflect the distances between probes positioned in a region external to the helix. Using the nucleoside analogues should give considerably different results since the measurement is being taken between points within the helix. An ongoing study is 
using 6-MI to function as an acceptor. The use of this pteridine nucleoside analogue will greatly improve the quality of the information that is available from this class of experiments.*

\subsection{2-Amino Purine}

\subsubsection{Background}

The potential for using fluorescent nucleoside analogues in DNA research was suggested in an article in 1969 by Ward et al. ${ }^{34}$ In this paper the fluorescence characteristics of 2-amino purine (2-AP) were defined, demonstrating that it would be practical for study because of a number of qualities. A quantum yield of 0.68 in an aqueous solvent at $\mathrm{pH} 7.0$ and an emission maximum of $370 \mathrm{~nm}$ identify this compound as a powerful tool for studies of DNA. These authors also successfully incorporated 2-AP into oligonucleotides using Escherichia coli RNA polymerase and substituting 2-AP-triphosphate for ATP. The fluorescence changes seen upon incorporation into an oligonucleotide made it apparent that this probe could be used to monitor changes in an oligonucleotide structure or environment. The synthesis of 2-AP as a phosphoramidite by McLaughlin et al. in $1988^{35}$ has allowed the site-specific incorporation of this fluorophore into oligonucleotides through standard automated synthesis. Since that time 2-AP has been used in various studies. Its behavior in oligonucleotides is similar to that of the pteridine nucleoside analogues in that it is quenched upon incorporation and fluorescence properties change dramatically when events or other molecules impact the structure of the oligonucleotide. Several examples of research using this probe are briefly described.

\subsubsection{Applications}

In a structural study of DNA containing 2-AP Nordlund et al have analyzed the movement and flexibility of DNA. ${ }^{4}$ This study utilized the temperaturedependent time-resolved fluorescence of 2-AP. By comparison of the single exponential decay of the monomeric probe and the multiexponential decay of the

*Deoxyribose, ribose, and phosphoramidite forms of the pteridines, 3-MI, 6-MI, and 6-MAP are available through Toronto Research Chemicals, Inc. (TRC), Toronto, Canada. Custom oligonucleotides containing 3-MI, 6-MI, and 6-MAP are available through TriLink BioTechnologies, Inc., San Diego, CA. 
probe within the oligonucleotide, conclusions could be drawn about conformational states of the probe. Data from this study leads to the conclusion that 2-AP within the oligonucleotide exists in four or more conformational states. These can be described as various levels of base stacking or unstacking within the helix.

Another study of structural dynamics of DNA was conducted by Guest et al. ${ }^{5}$ in which the strength of base-pairing interactions in various mismatches was examined. This study analyzed internal rotation of 2-AP within a double strand using fluorescence amplitude and correlation times. The degree of annealing of the two oligonucleotides was studied using tumbling correlation times and the emission lifetimes were used to determine the excited-state quenching of 2-AP. The findings demonstrate the usefulness of 2-AP to measure differences in the strength of base-pairing interactions as a function of base motion.

In a similar study by Hochstrasser et al. $^{2}$ 2-AP was used to label substrates for monitoring base-pairing interactions and local melting of DNA by measuring fluorescence decay characteristics. Once again, the complexity of the decay curve between a 2-AP-containing single strand and a 2-AP-containing double strand was used to draw conclusions about the single-strandedness of the 2-AP position. Variations in the apparent fraction of paired 2-AP bases led the authors to conclude that the Klenow fragment can melt a DNA duplex terminus resulting in a single-stranded conformation.

In a very simple and direct application of 2-AP for monitoring helicase activity Raney et al. ${ }^{3}$ used an oligonucleotide substrate containing seven 2-AP's spaced at regular intervals. Fluorescence intensity which is quenched 2-fold within the oligonucleotide is restored when the substrate is unwound by helicase. By measuring fluorescence intensity changes over real time, the rate of helicase driven unwinding of DNA was measured.

Frey et al. ${ }^{36}$ used 2-AP in a stopped flow study to examine the kinetics of the polymerization reaction of Klenow fragment. This experiment also utilizes the quenching affect of the double-strand conformation on 2-AP.

The measurement of very subtle movements between DNA and protein is presented in a paper by Beechem et al. ${ }^{37}$ In this study 2-AP was site-specifically incorporated into a primer terminus and then changes in fluorescence were observed as the T4 polymerase was allowed to bind. This protein has distinctly different binding sites for polymerase and exonuclease activities. A variety of factors can shift the predominant binding from one site to the other. After determining that fluorescence quenching or hyperfluorescence were dependent on the site to which the primer was bound, the authors were able to investigate transitions between polymerase site and exonuclease site bound primer. This is a good demonstration of the way the fluorophore can reflect subtle changes in its environment.

It is apparent from the number and diversity of applications using 2-AP, only 
a small sampling of which are noted here, that there are many ways to observe the changes experienced by the probe when changes occur in the oligonucleotide environment.

\subsection{1,N6-Ethenoadenosine}

Another potentially useful fluorescent nucleoside analogue is $1, \mathrm{~N}^{6}$-ethenoadenosine. This compound along with ethenocytidine have been used in research since their development in 1972. The fluorescence quantum yield of 0.5 and the maximum emission wavelength of $410 \mathrm{~nm}$ make ethenoadenosine a particularly attractive probe. ${ }^{38,39}$ A great deal was learned about the fluorescence characteristics of chloroacetaldehyde-modified dinucleosides. By exposing dinucleosides to chloroacetaldehyde under specific conditions a series of dinucleotides were modified to contain ethenoadenosine or ethenocytidine. Fluorescence analysis of the resulting compounds revealed that the fluorescence was quenched from the interactions with neighboring bases. It was apparent from these experiments that neighboring purines resulted in a greater quench than neighboring pyrimidines. ${ }^{40}$ Now that these probes have been synthesized as phosphoramidite forms allowing incorporation into DNA on an automated synthesizer, ${ }^{41}$ more can be learned about their behavior within the oligonucleotide strand. Since the etheno-compounds are unstable under standard deprotecting methods, it is necessary to use fast base deprotecting groups and a short treatment with ammonia.

\subsection{Summary and Outlook}

The focus of this chapter was to point out the unique properties of fluorescent nucleoside analogue probes and to examine how one can take advantage of those properties to observe quite subtle interactions within DNA. There are a growing number of nucleoside analogues that are highly fluorescent, stable, and available as phosphoramidites to facilitate site-specific incorporation into oligonucleotides. Some of these probes apparently participate in base pairing (2-AP, 6-MI, 6-MAP) and some are comparable to a single-base-pair mismatch as measured by melting temperatures. All four of the pteridine analogues, when incorporated into an oligonucleotide migrate through a polyacrylamide gel at a rate indistinguishable from the equivalent sequence as a control. This would suggest that there are no gross configurational changes in the oligonucleotide due to the presence of the pteridine. Table 5.5 lists some of the fluorescence properties of several probes for comparison. 
Table 5.5. Fluorescence Properties of Some Nucleoside Analogues

\begin{tabular}{lccclccc}
\hline Probe & $\mathrm{Ex}_{\max }$ & $\mathrm{Em}_{\max }$ & $\mathrm{Q}_{\text {rel }}$ & Probe & $\mathrm{Ex}_{\max }$ & $\mathrm{Em}_{\max }$ & $\mathrm{Q}_{\text {rel }}$ \\
\hline 3-MI & $348 \mathrm{~nm}$ & $431 \mathrm{~nm}$ & 0.88 & 6-MI & $340 \mathrm{~nm}$ & $430 \mathrm{~nm}$ & 0.70 \\
6-MAP & $330 \mathrm{~nm}$ & $435 \mathrm{~nm}$ & 0.39 & 2-AP & $303 \mathrm{~nm}$ & $370 \mathrm{~nm}$ & 0.68 \\
DMAP & $330 \mathrm{~nm}$ & $437 \mathrm{~nm}$ & 0.48 & Etheno-A & $300 \mathrm{~nm}$ & $410 \mathrm{~nm}$ & 0.50 \\
\hline
\end{tabular}

Studies using nucleoside analogues in oligonucleotides have demonstrated that subtle changes in DNA often can be observed using a variety of fluorescence properties. They provide us with a new window on DNA structure and its relationship with the environment. In many cases the probes can be placed so close to the site of interest that changes at that site can be witnessed in a real time format using various fluorescence techniques.

The ideal probe, one that closely resembles the natural bases and is highly fluorescent, however, is quite elusive. As with most things in science, the problem is much more complex than one might expect. The characteristics that make guanosine recognizable from the point of view of a protein, for example, extend beyond the hydrogen bonding moieties. The size, shape, and electronic distribution within guanosine all contribute to its being recognized. Nothing can mimic this perfectly without being guanosine and consequently being only faintly fluorescent. The objective is to create the most native-like, stable and highly fluorescent probes possible. In the search for an ideal analogue, something must always be sacrificed in order to achieve acceptable levels of stability and fluorescence. There is substantial structural difference between fluorescent nucleoside analogues and native nucleosides, and yet similarities in overall shape, size, and linkage allow us to place a fluorophore within the DNA without totally disrupting the tertiary structure. With a variety of different analogues available, it will be possible to select the one that is most appropriate for a given application.

The difference between the conventional fluorophores and the analogues is only a matter of subtlety. There is no dispute about the potential and usefulness of the hundreds of conventional fluorophores now available for research. Having a variety of analogues capable of being placed within in a specific sequence and at different sites will give researchers one more way to approach the study of DNA.

\section{Acknowledgments}

I would like to thank Dr. Ishita Mukerji and Kristi Wojtuszewski at Wesleyan University for sharing their research on the HU protein; Basil Hanss at Mt. Sinai 
for his contribution on oligonucleotide transport; and Dr. William Laws and Ed Radhofsky for their contribution on DNA conformation. I also wish to thank Drs. Jay Knutson and Frank Balis for helpful comments and stimulating discussions.

\section{References}

1. Hawkins, M. E., Pfleiderer, W., Mazumder, A., Pommier, Y. G., and Balis, F. M. Nucleic Acids Res. 23, 2872-2880, 1995.

2. Hochstrasser, R. A., Carver, T. E., Sowers, L. C. and Millar, D. P. Biochemistry, 33, 11971-11979, 1994.

3. Raney, K. D., Sowers, L. C., Millar, D. P., and Benkovic, S. J. Proc. Natl. Acad. Sci. USA 91, 6644-6648, 1994.

4. Nordlund, T. M., Wu, P., Andersson, S., Nilsson, L., Rigler, R., Graslund, A., McLaughlin, L. W., and Gildea, B. SPIE Time-Resolved Laser Spectrosc. Biochem. II 1204, 344-353, 1990.

5. Guest, C. R., Hochstrasser, R. A., Sowers, L. C., and Millar, D. P. Biochemistry 30, 3271-3279, 1991.

6. Hawkins, M. E., Pfleiderer, W., Balis, F. M., Porter, D., and Knutson, J. R. Anal. Biochem. 244, 86-95, 1997.

7. Hawkins, M. E., Pfleiderer, W., Jungmann, O., and Balis, F. Anal. Biochem. 298, 231-240, 2001.

8. Driscoll, S. L., Hawkins, M. E., Balis, F. M., Pfleiderer, W., and Laws, W. R. Biophys. J. 73, $3277-$ 3286, 1997.

9. Davenport, L., Knutson, J. R., and Brand, L. Faraday Discuss. Chem. Soc. 81, 81-94, 1986.

10. Brown, P. O., Bowerman, B., Varmus, H. E., and Bishop, J. M. Cell 49, 347-356, 1987.

11. Brown, P. O., Bowerman, B., Varmus, H. E. and Bishop, J. M. Proc. Natl. Acad. Sci. USA 86, 2525-2529, 1989.

12. Fujiwara, T., and Craigie, R. Proc. Natl. Acad. Sci. USA 86, 3065-3069, 1989.

13. Vink, C., Banks, M., Bethell, R., and Plasterk, R. H. A. Nucleic Acids Res. 22, 2176-2177, 1994.

14. Katzman, M., Katz, R. A., Skalka, A. M., and Leis, J. J. Virol. 63, 5319-5327, 1989.

15. Wojtuszewski, K., Hawkins, M. E., Cole, J. L., and Mukerji, I. Biochemistry 40, 2588-2598, 2001.

16. Broyles, S. S., and Pettijohn, D. E. J. Mol. Biol. 187, 47-60, 1986.

17. Bonnefoy, E., and Rouviere-Yaniv, J. EMBO J. 10, 687-696, 1991.

18. Rice, P. A., Yang, S., Mizuuchi, K., and Nash, H. A. Cell 87, 1295, 1996.

19. Hodges-Garcia, Y., Hagerman, P. J., and Pettijohn, D. E. J. Biol. Chem. 264, 14621-14623, 1989.

20. Cole-Strauss, A., Yoon, K., Xiang, Y., Byrne, B. C., Rice, M. C., Gryn, J., Holloman, W. K. and Kmiec, E. B. Science 273, 1386-1389, 1996.

21. Cossum, P. A., Sasmor, H., Dellinger, D., Truong, L., Cummins, L., Owens, S. R., Markham, P. M., Shea, J. P., and Crooke, S. J. Pharmacol. Exp. Ther. 267, 1181-1190, 1993.

22. Meeker, R., Legrand, G., Ramirez, J., Smith, T., and Shih, Y. H. J. Neuroendocrinol. 7, 419-428, 1995.

23. Oberbauer, R., Schreiner, G. F., and Meyer, T. W. Kidney Int. 48, 1226-1232, 1995.

24. Ogawa, S., Brown, H. E., Okano, H. J., and Pfaff, D. W. Regul. Pept. 59, 143-149, 1995.

25. Rappaport, J., Hanss, B., Kopp, J. B., Copeland, T. D., Bruggeman, L. A., Coffman, T. M., and Klotman, P. E. Kidney Int. 47, 1462-1469, 1995.

26. Yakubov, L. A., Deeva, E. A., Zarytova, V. F., Ivanova, E. I., Ryte, A. S., Yurchenko, L. V., and Vlassov, V. V. Proc. Natl. Acad. Sci. USA 86, 6454-6458, 1989.

27. Wu-Pong, S., Weiss, T. L., and Hunt, C. A. Cell. Mol. Biol. 40, 843-850, 1994.

28. Iverson, P. L., Zhu, S., Meyer, A., and Zon, G. Antisense Res. Dev. 2, 221-222, 1992. 
29. Crooke, R. M., Graham, M. F., Cooke, M. E. and Crooke, S. T. J. Pharmacol. Exp. Ther 275, 462473, 1995.

30. Zhao, Q., Waldschmidt, T., Fisher, E., Herrera, C. J., and Krieg, A. M. Blood 84, 3660-3666, 1994.

31. Parkhurst, K. M., Brenowitz, M., and Parkhurst, L. J. Biochemistry 23, 7459-7465, 1996.

32. Parkhurst, K. M., and Parkhurst, L. J. Biochemistry 34, 293-300, 1995.

33. Parkhurst, K. M., and Parkhurst, L. J. Biochemistry 34, 285-292, 1995.

34. Ward, D. C., and Reich, E. J. Biol. Chem. 244, 1228-1237, 1969.

35. McLaughlin, L. W., Leong, T., Benseler, F., and Piel, N. Nucleic Acids Res. 16, 5631-5644, 1988.

36. Frey, M. W., Sowers, L. C., Millar, D. P., and Benkovic, S. J. Biochemistry 34, 9185-9192, 1995.

37. Beecham, J. M., Otto, M. R., Bloom, L. B., Eritja, R., Reha-Krantz, L. J., and Goodman, M. F. Biochemistry 37, 10144-10155, 1998.

38. Leonard, N. J. CRC Crit. Rev. Biochem. 15, 125-199, 1993.

39. Secrist, J. A., Barrio, J. R., and Leonard, N. J. Science 175, 646-647, 1972.

40. Tolman, G. L., Barrio, J. R., and Leonard, N. J. Biochemistry 13, 4869, 1974.

41. Srivastava, S. C., Raza, S. K., and Misra, R. Nucleic Acids Res. 22, 1296-1304, 1994.

42. Hawkins, M. E. Cell Biochem. Biophys. 34, 257-281, 2001. 